\title{
Progress in research on sintering and microstructural development
}

\author{
Suk-Joong L. Kang • Rajendra Bordia • \\ Didier Bouvard · Eugene Olevsky
}

Received: 14 June 2012/ Accepted: 14 June 2012/Published online: 18 July 2012

(C) Springer Science+Business Media, LLC 2012

This special section of the Journal of Materials Science includes a number of full-length articles based on research presented at the International Conference on Sintering 2011, which was held on Jeju Island, Republic of Korea, on August 26-September 1, 2011. The meeting was chaired by Professors Suk-Joong L. Kang, Rajendra K. Bordia, Didier Bouvard, and Eugene Olevsky, who are also the Guest Editors for this special section.

The Jeju Island meeting was the sixth in a series that started in 1995 as a continuation of the well-known cycle of conferences on sintering and related phenomena organized by G. Kuczynski that ran from 1967 to 1983 . The first five meetings in this reestablished series of conferences were held at Pennsylvania State University in 1995, 1999 and 2003; in Grenoble, France in 2005; and in San Diego, California in 2008.

\section{S.-J. L. Kang}

Department of Materials Science \& Engineering, Korea

Advanced Institute of Science and Technology,

Daejeon 305-701, Republic of Korea

e-mail: sjkang@kaist.ac.kr

\section{R. Bordia ( $\square)$}

Department of Materials Science and Engineering Roberts Hall, University of Washington, Seattle, Box 352120, WA, USA

e-mail: bordia@u.washington.edu

\section{Bouvard}

Institut Polytechnique de Grenoble Laboratoire SIMAP - GPM2, BP 46, Saint Martin d'Hères 38402, France

e-mail: didier.bouvard@grenoble-inp.fr

\section{E. Olevsky}

Department of Mechanical Engineering College of Engineering, San Diego State University, 5500 Campanile Drive, San Diego 92182-1323, CA, USA

e-mail: eolevsky@mail.sdsu.edu
Sintering 2011 brought together more than 260 participants from 27 countries, fostering a high level of scientific interaction and creating an atmosphere of broad international collaboration. The meeting included participants from North and Central America, Europe (both Eastern and Western), Asia, Australia and Africa.

The conference demonstrated the advances that have been made in the areas of the multi-scale modeling of densification and in microstructure development and promoted a better understanding of the processing of complex systems (multi-layered, composite and reactive systems). Concerning sintering technology, innovative approaches, such as field-assisted sintering attracted the attention of the materials processing community. Other timely topics included the sintering and microstructural development of nanostructured materials, and the sintering of bio- and energy applications-related materials.

This collection of articles published by the Journal of Materials Science covers the rich diversity of the sintering science and technology topics presented at the conference. Both the conference participants and organizers had to meet numerous deadlines to enable the timely publication of this special issue of the Journal of Materials Science.

We hope that this collection of articles will be an important contribution to the literature, and we are looking forward to seeing you at future Sintering conferences. 